\title{
Perturbative behavior of a vortex in a trapped Bose-Einstein condensate
}

\author{
Lyndon Koens and Andrew M. Martin \\ School of Physics, The University of Melbourne, Parkville 3010, Australia
}

(Received 29 February 2012; published 5 July 2012)

\begin{abstract}
We derive a set of equations that describes the shape and behavior of a single perturbed vortex line in a Bose-Einstein condensate. Through the use of a matched asymptotic expansion and a unique coordinate transform, a relation for a vortex's velocity, anywhere along the line, is found in terms of the trapping, rotation, and distortion of the line at that location. This relation is then used to find a set of differential equations that give the line's specific shape and motion. This work extends a previous similar derivation by Svidzinsky and Fetter [Phys. Rev. A 62, 063617 (2000)], and enables a comparison with recent numerical results.
\end{abstract}

DOI: 10.1103/PhysRevA.86.013605

PACS number(s): 03.75.Kk, 03.65.-w, 05.30.Jp

\section{INTRODUCTION}

Experimental realizations of vortices in Bose-Einstein condensates (BECs) [1-5] has spurred much research into the behavior and effects that vortices have on a BEC [6,7]. Similar to the vortices that Feynman predicted in superfluid He II [8], vortices in BECs have quantized circulation, ensuring that the condensate phase is a single-valued function (see Ref. [9] for details). However, unlike in superfluid He II, the vortices in a $\mathrm{BEC}$ are sufficiently described in the mean-field regime by the Gross-Pitaevskii equation [10,11] with rotation [Eq. (1)],

$$
\begin{aligned}
& {\left[-\frac{\hbar^{2}}{2 M} \nabla^{2}+V_{t r}(\mathbf{r})+g|\Psi|^{2}-\mu(\Omega)\right] \Psi} \\
& +i \hbar \boldsymbol{\Omega} \cdot(\mathbf{r} \times \nabla) \Psi=i \hbar \frac{\partial \Psi}{\partial t}
\end{aligned}
$$

where $\Psi$ is the condensate wave function, $V_{t r}(\mathbf{r})$ is the external trapping potential, $\boldsymbol{\Omega}$ is the condensate rotation vector, $g=$ $4 \pi a \hbar^{2} / M$ is the interparticle interaction strength, $\mu(\Omega)$ is the chemical potential, $M$ is the particle mass, and $a$ is the $s$-wave scattering length.

In a stationary condensate, it is energetically unfavorable to contain vortices; however, when $\Omega>\Omega_{c}=$ $5 \hbar \ln \left(R_{\perp} / r_{c}\right) / 2 M R_{\perp}^{2}$ (with $R_{\perp}$ being the radius of the BEC perpendicular to the rotation vector and $r_{c}$ being the vortex core radius), a centered vortex becomes energetically favorable [12]. Upon investigating the dissipation of such a vortex, the vortex's dissipation time was shown to depend on the condensate's temperature [13].

In a trapped BEC, a quantized vortex is rarely stationary, usually moving or contorting. An off-centered straight-line vortex is known to precess at $\dot{\phi}=3 \Omega_{c} / 5\left(1-\rho_{0}^{2} / R_{\perp}^{2}\right.$ ) (with $\rho_{0}$ being the cylindrical radial coordinate of the vortex line) $[12,14]$. This straight structure is representative of a vortex in a pancake-shaped condensate, $R_{\perp} / R_{z}>1$, where $R_{z}$ is the condensate radius parallel to the rotation vector. For a cigar-shaped trap, $R_{\perp} / R_{z}<1$; however, the vortex line bends. This result was shown numerically in Refs. [15,16], seen experimentally by Rosenbusch et al. [17], and reasoned physically by Modugno et al. [18]. A quantized vortex also supports wave perturbations, coupling with and modifying the normal modes of the condensate $[19,20]$ or supporting helical-wave structures, such as Kelvin waves [21], along its length $[10,22]$.
With such a wide range of behavior, a single set of equations that completely describes the vortex has been desired. Early attempts to derive this equation relied on the method of matched asymptotic expansion [23,24]. This procedure was extended by Svidzinsky and Fetter [25] to produce a set of equations that should generally describe all small perturbations from a straight-line vortex. This derivation used a coordinate transform to account for vortex line bending; however, in its execution, these coordinates were not formally defined, and the outer and inner solutions matched through a pseudo "vector potential." This gave rise to a modified set of equations to those that describe the vortex's motion and structure.

This paper rederives this procedure to determine the behavior and structure of a slightly perturbed straight-line vortex, using coordinate transformations and the method of matched asymptotic expansion. Section II justifies the relevance and procedure of such an expansion, and Sec. III defines the unique coordinate system used. An inner and outer solution are then found (Secs. IV and V, respectively) and matched (Sec. VI) to give a relation between the perturbed vortex's motion and shape. This relation contains an unknown constant that is determined by comparing to known physical scenarios (Sec. VII). This then allows simple results from the relation to be calculated and compared to numerical simulations (Sec. VIII). Finally, in Sec. IX, a general comparison between this work and Ref. [25] is performed, illustrating the differences in methodology and general results.

\section{THE IDEA}

BECs with vortices within them have two natural length scales: the condensate length scale $R_{\perp}$ and the vortex core radius $r_{c}$, with $r_{c}<R_{\perp}$. These two scales suggest that a matched asymptotic expansion [26] can be performed to determine the behavior near the vortex core, length scale $r_{c}$, and far from the vortex core, length scale $R_{\perp}$, and matched to give the system's full behavior. Such an asymptotic expansion has been previously preformed for vortices in simplified scenarios in Refs. [23] and [24].

In the local coordinates of the vortex line, the inner solution depends on properties of the vortex line, which when matched to the outer solution creates a relation between the shape and velocity of the line. This relationship then gives a set of 


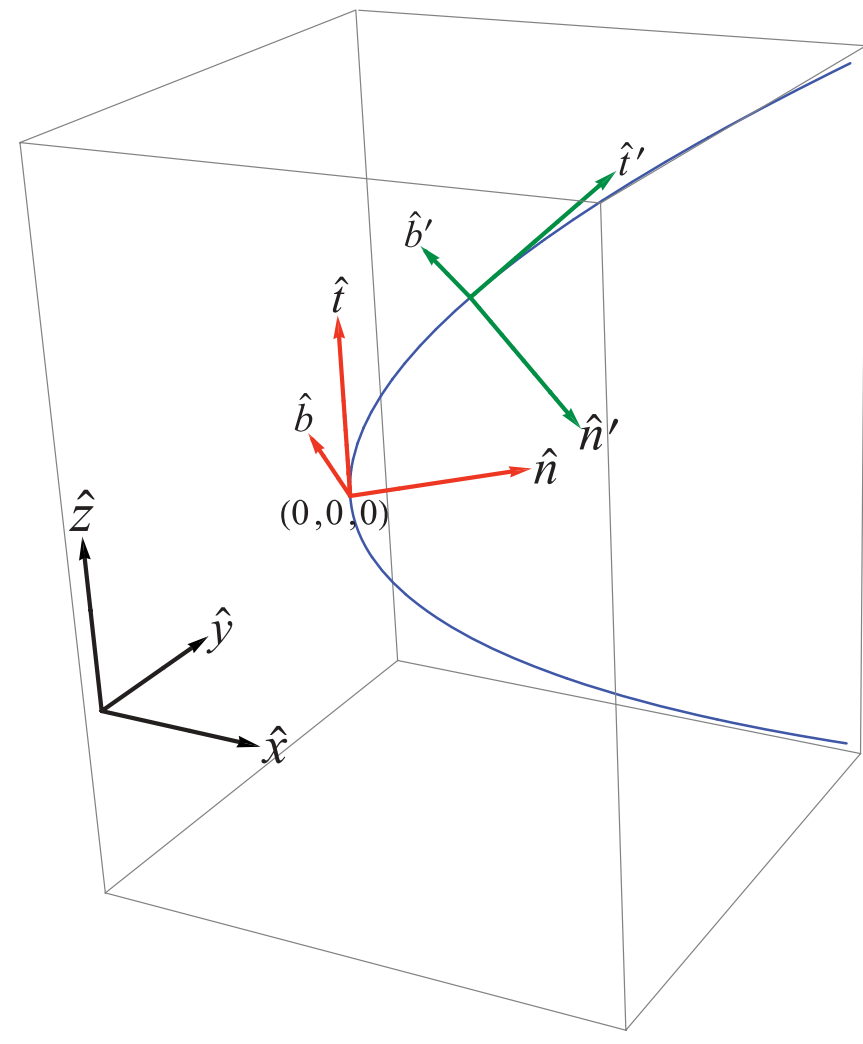

FIG. 1. (Color online) Depiction of the local coordinate vectors $\hat{\mathbf{t}}, \hat{\mathbf{n}}$, and $\hat{\mathbf{b}}$ of the curve $\mathbf{c}(T)=\left\{T^{2} / 2, T^{2} / 2, T\right\}$, with the coordinate vectors plotted for $T=0$ (red, lower set) and $T=1$ (green, upper set).

differential equations that describes the vortex line's structure and motion through space.

\section{THE LOCAL COORDINATES OF THE VORTEX LINE}

In a BEC, the localized vorticity from a vortex creates a single vortex line. Each point on this line has a position and a velocity; therefore, the vortex line, at a given time, is described by two parametrized functions: a curve describing the line's shape and position, $\mathbf{c}(T)$, and a velocity vector, $\mathbf{V}(T)$. This parametrization allows for the transformation into the local coordinate system, with the curve $\mathbf{c}(T)$ determining the axes and $\mathbf{V}(T)$ representing the line's motion at a given point.

For any curve $[\mathbf{c}(T)]$, a tangent vector [Eq. (2)], a normal vector [Eq. (3)], and a binormal vector [Eq. (4)] are defined; see Fig. 1. These three orthogonal vectors form the basis to the coordinate system of the curve, known as the Frenet-Serret coordinates [27],

$$
\begin{aligned}
\hat{\mathbf{t}} & =\frac{\mathbf{c}^{\prime}(T)}{\left|\mathbf{c}^{\prime}(T)\right|} \\
\hat{\mathbf{n}} & =\frac{\mathbf{c}^{\prime}(T) \times\left[\mathbf{c}^{\prime \prime}(T) \times \mathbf{c}^{\prime}(T)\right]}{\left|\mathbf{c}^{\prime}(T)\right|\left|\mathbf{c}^{\prime \prime}(T) \times \mathbf{c}^{\prime}(T)\right|}, \\
\hat{\mathbf{b}} & =\hat{\mathbf{t}} \times \hat{\mathbf{n}} .
\end{aligned}
$$

At a given $T$, such a basis is equivalent to the basis of a Cartesian coordinate system, allowing the respective $\hat{\mathbf{t}}, \hat{\mathbf{n}}$, and $\hat{\mathbf{b}}$ vectors to be locally treated as $\hat{\mathbf{z}}, \hat{\mathbf{x}}$, and $\hat{\mathbf{y}}$.
This local Cartesian behavior extends to the derivatives in the $\hat{\mathbf{n}}$ and $\hat{\mathbf{b}}$ directions. Translations in these directions do not change the basis vectors, and therefore the local derivatives in $\hat{\mathbf{n}}$ and $\hat{\mathbf{b}}$ are identical to their Cartesian counterparts $\left(\partial_{n} \equiv\right.$ $\partial_{x}, \partial_{n}^{2} \equiv \partial_{x}^{2}$, etc.).

The curve progresses in $\hat{\mathbf{t}}$ causing the local derivatives in $\hat{\mathbf{t}}$ to not match its Cartesian equivalent. Using the chain rule, this deviation can be quantified, showing the first-order derivatives in $\hat{\mathbf{t}}$ to be

$$
\left.\frac{\partial}{\partial T}\right|_{T=T_{0}}=\lim _{T \rightarrow T_{0}} \mathbf{c}^{\prime}(T) \cdot \nabla,
$$

from which higher-order derivatives can be constructed:

$$
\begin{aligned}
& \left.\frac{\partial}{\partial T}\left(\frac{\partial}{\partial T}\right)\right|_{T=T_{0}} \\
& \quad=\lim _{T \rightarrow T_{0}}\left[\mathbf{c}^{\prime \prime}(T) \cdot \nabla+\mathbf{c}^{\prime}(T) \otimes \mathbf{c}^{\prime}(T): \nabla \otimes \nabla\right],
\end{aligned}
$$

where $\otimes$ represents the outer product and : represents a double contraction.

In this case, the structure of the vortex line is unknown. Therefore, in order to evaluate these derivatives, a pseudoparametrization, which describes the local behavior and can take any shape, needs to be used.

Conveniently, any unique curve is defined through two terms: curvature [Eq. (7)] and torsion [Eq. (8)]. These terms describe how a curve bends and distorts through space, irrespective of its location and choice of parametrization. Curvature indicates how the curve bends in the $\hat{\mathbf{t}}-\hat{\mathbf{n}}$ plane (with $1 / \kappa$ being the radius of a circle at a given point) and torsion indicates how the curve twists out of the $\hat{\mathbf{t}}-\hat{\mathbf{n}}$ plane (how $\hat{\mathbf{n}}$ and $\hat{\mathbf{b}}$ rotate as $T$ progresses):

$$
\begin{aligned}
\kappa & =\frac{\left|\mathbf{c}^{\prime}(T) \times \mathbf{c}^{\prime \prime}(T)\right|}{\left|\mathbf{c}^{\prime}(T)\right|^{3}}, \\
\tau & =\frac{\left[\mathbf{c}^{\prime}(T) \times \mathbf{c}^{\prime \prime}(T)\right] \cdot \mathbf{c}^{\prime \prime \prime}(T)}{\left|\mathbf{c}^{\prime}(T) \times \mathbf{c}^{\prime \prime}(T)\right|^{2}} .
\end{aligned}
$$

Generally, a curve's torsion and curvature vary along its length, allowing for all possible three-dimensional (3D) curve structures to form. However, locally around any point the torsion and curvature are effectively constant, prompting the use of a modified helix for the pseudoparametrization of the vortex line:

$$
\mathbf{c}(T)=\{a \cos (T), a \sin (T), b T\} .
$$

Assuming the excitations on a vortex line are small compared with the vortex's overall structure, the curve's tangent vector will almost align with $\hat{\mathbf{z}}$. This condition can be enforced by adding appropriate linear terms to the parametrization to make $\left.\hat{\mathbf{t}}\right|_{T=0} \approx \hat{\mathbf{z}}$. This condition also implies that the length of the curve is predominantly the length traveled in $z$ ( $b$ is large); this simplifies the renormalization of the parametrization, from $T$ into the arc length of the curve $s \approx z=b T$. This is an easier parametrization to work with, as curves parametrized by arc length have $\left|\mathbf{c}^{\prime}(s)\right|=1$.

Furthermore, helices have no variation in $\rho$. Hence for the complete curve to take any form, a constant pseudocurvature $k$, between $\rho$ and $z$, needs to be added to the pseudoparametrization [with $k$ being defined as $\approx \sqrt{\rho^{\prime \prime}(s)^{2}+z^{\prime \prime}(s)^{2}}$ ]. Keeping 


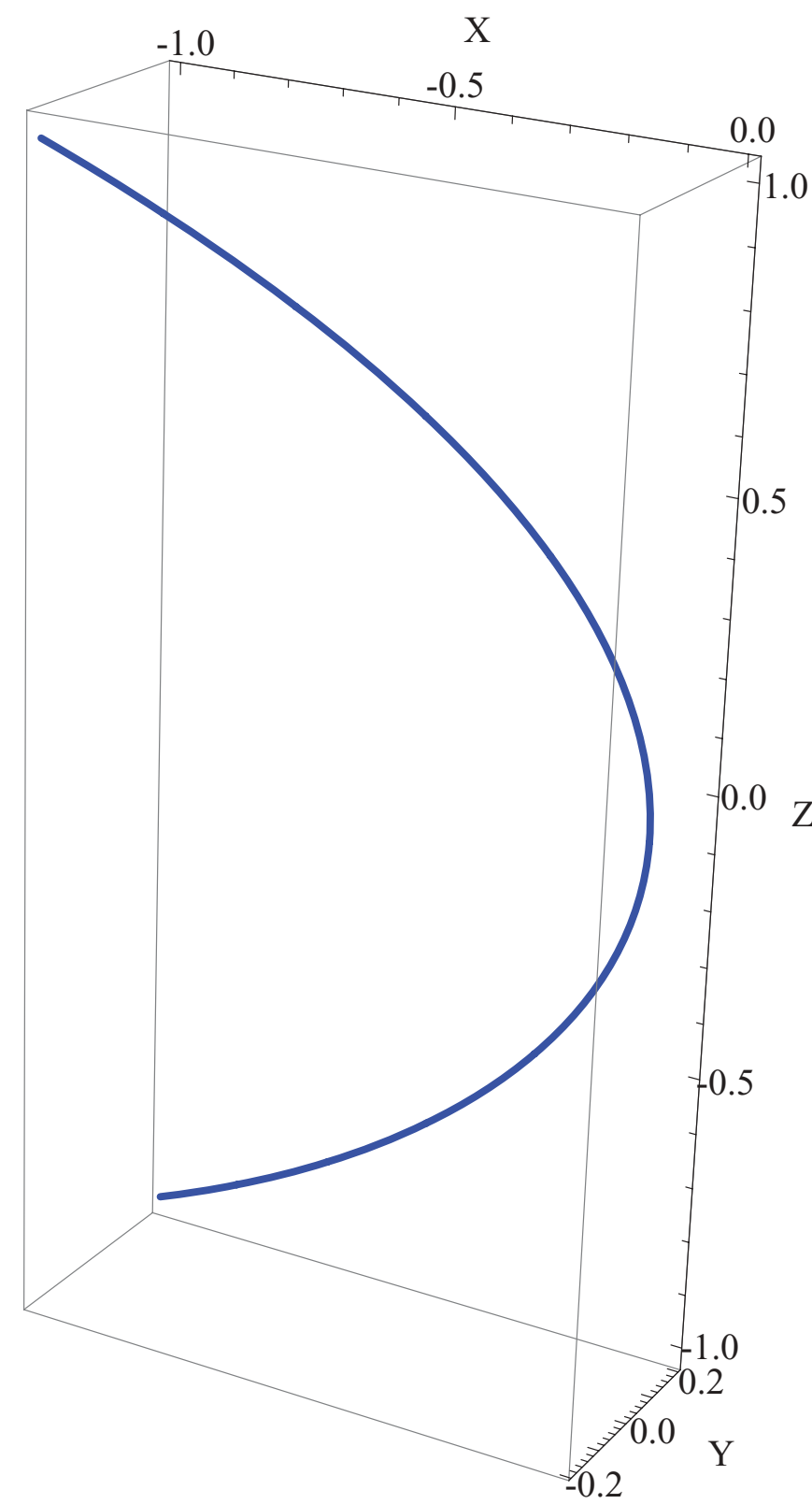

FIG. 2. (Color online) Plot of the pseudoparametrization used (dimensionless) [Eq. (10) with $a=1, k=2$, and $b=5$ ].

the $z(s)$ form $[z(s)=s], \rho^{\prime \prime}(s)$ equals $-k$. Hence the pseudoparametrization becomes (Fig. 2)

$$
\begin{aligned}
\mathbf{c}(s)= & \left\{\left(a-\frac{k}{2} s^{2}\right) \cos \left(\frac{s}{b}\right)+\alpha s,\right. \\
& \left.\left(a-\frac{k}{2} s^{2}\right) \sin \left(\frac{s}{b}\right)-\left(\frac{a}{b}-\beta\right) s, s\right\},
\end{aligned}
$$

where $\alpha$ and $\beta$ are the small angles of deviation from $\hat{\mathbf{z}}$ in the $\hat{\mathbf{x}}$ and $\hat{\mathbf{y}}$ directions, respectively, i.e., $\left.\hat{\mathbf{t}}\right|_{s=0}=\{\alpha, \beta, 1\}$.

This parametrization makes the curvature, torsion, and local derivatives in $s$, to the first order in $\alpha$ and $\beta$, to be

$$
\begin{aligned}
\kappa & =\frac{a}{b^{2}}+k, \\
\tau & =\frac{a+3 b^{2} k}{b\left(a+b^{2} k\right)}, \\
\frac{\partial}{\partial s} & \rightarrow \frac{\partial}{\partial z}+\alpha \frac{\partial}{\partial x}+\beta \frac{\partial}{\partial y},
\end{aligned}
$$

and

$$
\frac{\partial^{2}}{\partial s^{2}} \rightarrow \frac{\partial^{2}}{\partial z^{2}}-\kappa \frac{\partial}{\partial x}+2 \alpha \frac{\partial^{2}}{\partial x \partial z}+2 \beta \frac{\partial^{2}}{\partial y \partial z} .
$$

This makes the gradient and Laplacian operators $\left(\nabla^{\prime}\right.$ and $\left.\nabla^{\prime 2}\right)$

$$
\nabla^{\prime}=\nabla+\left(\alpha \frac{\partial}{\partial x}+\beta \frac{\partial}{\partial y}\right) \hat{\mathbf{z}},
$$

and

$$
\nabla^{\prime 2}=\nabla^{2}-\kappa \frac{\partial}{\partial x}+2 \alpha \frac{\partial^{2}}{\partial x \partial z}+2 \beta \frac{\partial^{2}}{\partial y \partial z},
$$

where $\nabla$ has the standard Cartesian definition $\left(\nabla=\left\{\partial_{x}, \partial_{y}, \partial_{z}\right\}\right)$.

Using these to transform the Gross-Pitaevskii equation [Eq. (1)] into the local coordinates around a point on the vortex line $\mathbf{r}_{\mathbf{0}}$, assuming that $\boldsymbol{\Omega}$ is in $\hat{\mathbf{z}}$, and assuming that the vortex line has no velocity in $\hat{\mathbf{z}}$, the equation for the behavior near the vortex core becomes

$$
\begin{aligned}
- & \frac{\hbar^{2}}{2 M}\left(\nabla^{2}-\kappa \frac{\partial}{\partial x}+2 \alpha \frac{\partial^{2}}{\partial x \partial z}+2 \beta \frac{\partial^{2}}{\partial y \partial z}\right) \Psi \\
& +V_{t r}\left(\mathbf{r}_{\mathbf{0}}\right) \Psi+g|\Psi|^{2} \Psi-\mu(\Omega) \Psi \\
& +\Psi \mathbf{r} \cdot\left[\nabla+\left(\alpha \frac{\partial}{\partial x}+\beta \frac{\partial}{\partial y}\right) \hat{\mathbf{z}}\right] V_{t r}\left(\mathbf{r}_{\mathbf{0}}\right) \\
& +i \hbar\left(\boldsymbol{\Omega} \times \mathbf{r}_{\mathbf{0}}\right) \cdot \nabla \Psi=i \hbar\left(\frac{\partial \Psi}{\partial t}-\mathbf{V} \cdot \nabla \Psi\right),
\end{aligned}
$$

where the trap terms have been expanded to the first order (order $r_{c}$ ), $\mathbf{r}$ is the position vector in the local coordinate system, $\boldsymbol{\Omega}$ is the rotation vector, and $\mathbf{V}$ is the velocity of the vortex line at $\mathbf{r}_{\mathbf{0}}$.

This equation describes the behavior of the condensate near the vortex core in terms of the properties of the vortex core itself. Hence, solving this equation gives the desired innerregion behavior of the $\mathrm{BEC}$.

\section{THE BEHAVIOR NEAR THE VORTEX CORE}

The steady-state solutions of Eq. (17) describe a condensate containing a vortex with standing waves along the line of the vortex. Hence to determine a relation for these standing waves, the inner equation to solve is

$$
\begin{aligned}
& -\frac{\hbar^{2}}{2 M}\left(\nabla^{2}-\kappa \frac{\partial}{\partial x}+2 \alpha \frac{\partial^{2}}{\partial x \partial z}+2 \beta \frac{\partial^{2}}{\partial y \partial z}\right) \Psi \\
& +V_{t r}\left(\mathbf{r}_{\mathbf{0}}\right) \Psi+g|\Psi|^{2} \Psi-\mu(\Omega) \Psi \\
& +\Psi \mathbf{r} \cdot\left[\nabla+\left(\alpha \frac{\partial}{\partial x}+\beta \frac{\partial}{\partial y}\right) \hat{\mathbf{z}}\right] V_{t r}\left(\mathbf{r}_{\mathbf{0}}\right) \\
& =-i \hbar \mathbf{V}^{\prime} \cdot \nabla \Psi,
\end{aligned}
$$

where $\mathbf{V}^{\prime}=\mathbf{V}+\left(\boldsymbol{\Omega} \times \mathbf{r}_{\mathbf{0}}\right)$. 
The small nature of the wave perturbations gives four small parameters (assumed to be of similar order) to use for a perturbation expansion: $\kappa, \alpha, \beta$, and $r_{c}$. The zeroth-order terms of this expansion describe a condensate with an unperturbed vortex,

$$
\left[-\frac{\hbar^{2}}{2 M} \nabla^{2}+V_{t r}\left(\mathbf{r}_{\mathbf{0}}\right)+g\left|\Psi_{0}\right|^{2}-\mu(\Omega)\right] \Psi_{0}=0 .
$$

The first-order terms account for the perturbations on the vortex line,

$$
\begin{aligned}
- & \frac{\hbar^{2}}{2 M} \nabla^{2} \Psi_{1}+V_{t r}\left(\mathbf{r}_{\mathbf{0}}\right) \Psi_{1}+2 g\left|\Psi_{0}\right|^{2} \Psi_{1}+g \Psi_{0}^{2} \Psi_{1}^{*}-\mu(\Omega) \Psi_{1} \\
= & -\frac{\hbar^{2} \kappa}{2 M} \frac{\partial \Psi_{0}}{\partial x}+\frac{\hbar^{2} \alpha}{M} \frac{\partial^{2} \Psi_{0}}{\partial x \partial z}+\frac{\hbar^{2} \beta}{M} \frac{\partial^{2} \Psi_{0}}{\partial y \partial z} \\
& -i \hbar \mathbf{V}^{\prime} \cdot \nabla \Psi_{0}-\Psi_{0} \mathbf{r} \cdot\left[\nabla+\left(\alpha \frac{\partial}{\partial x}+\beta \frac{\partial}{\partial y}\right) \hat{\mathbf{z}}\right] V_{t r}\left(\mathbf{r}_{\mathbf{0}}\right) .
\end{aligned}
$$

Only the far-from-core behavior of the inner solution is used for matching. Hence, the large $\rho=\sqrt{x^{2}+y^{2}}$ behavior of Eq. (20) is required. In this regime, $\left|\Psi_{0}\right|$ should be cylindrically symmetric, and can be approximated by the Thomas-Fermi solution $\left|\Psi_{T F}\right| \approx \sqrt{\left[\mu(\Omega)-V_{t r}\left(\mathbf{r}_{\mathbf{0}}\right)\right] / g}$. This simplifies Eq. (20) to

$$
\begin{aligned}
- & \frac{\hbar^{2}}{2 M} \nabla^{2} \Psi_{1}+g\left|\Psi_{0}\right|^{2} \Psi_{1}+g \Psi_{0}^{2} \Psi_{1}^{*} \\
= & -\Psi_{0} z \partial_{z^{\prime}} V_{t r}\left(\mathbf{r}_{\mathbf{0}}\right)+\cos (\phi)\left[\frac{\hbar^{2} \alpha}{M} \frac{\partial^{2} \Psi_{0}}{\partial \rho \partial z}-\Psi_{0} \rho\left|\nabla V_{t r}\left(\mathbf{r}_{\mathbf{0}}\right)\right|_{x}\right. \\
& \left.+\frac{\hbar^{2} \beta}{M \rho} \frac{\partial^{2} \Psi_{0}}{\partial \phi}-\frac{i \hbar V_{y}^{\prime}}{\rho} \frac{\partial \Psi_{0}}{\partial \phi}-\left(\frac{\hbar^{2} \kappa}{2 M}+i \hbar V_{x}^{\prime}\right) \frac{\partial \Psi_{0}}{\partial \rho}\right] \\
& +\sin (\phi)\left[\frac{\hbar^{2} \beta}{M} \frac{\partial^{2} \Psi_{0}}{\partial \rho}-\Psi_{0} \rho\left|\nabla V_{t r}\left(\mathbf{r}_{\mathbf{0}}\right)\right|_{y}-\frac{\hbar^{2} \alpha}{M \rho} \frac{\partial^{2} \Psi_{0}}{\partial \phi \partial z}\right. \\
& \left.-i \hbar V_{y}^{\prime} \frac{\partial \Psi_{0}}{\partial \rho}+\frac{1}{\rho}\left(\frac{\hbar^{2} \kappa}{2 M}+i \hbar V_{x}^{\prime}\right) \frac{\partial \Psi_{0}}{\partial \phi}\right],
\end{aligned}
$$

where $\partial_{z^{\prime}}=\partial_{z}+\alpha \partial_{x}+\beta \partial_{y}$, and $\left|\nabla V_{t r}\left(\mathbf{r}_{\mathbf{0}}\right)\right|_{i}$ and $V_{i}^{\prime}$ are the trap gradient and vortex line velocity, respectively, in the $i$ direction.

Assuming that the wave function has the form

$$
\begin{aligned}
\Psi= & {\left[\left|\Psi_{0}\right|+D(\rho, z)+\chi(\rho, z) \cos (\phi)\right.} \\
& +\zeta(\rho, z) \sin (\phi)] e^{i q \phi+i \eta(\rho, z) \cos (\phi)+i \lambda(\rho, z) \sin (\phi)}
\end{aligned}
$$

makes $\Psi_{0}=\left|\Psi_{0}\right| e^{i q \phi}$ and

$$
\begin{aligned}
\Psi_{1}= & {[D(\rho, z)+\chi(\rho, z) \cos (\phi)} \\
& +\zeta(\rho, z) \sin (\phi)+i\left|\Psi_{0}\right| \eta(\rho, z) \cos (\phi) \\
& \left.+i\left|\Psi_{0}\right| \lambda(\rho, z) \sin (\phi)\right] e^{i q \phi},
\end{aligned}
$$

where $q$ is the winding number of the vortex.

This reduces Eq. (21) into five coupled differential equations describing the behavior of the perturbation functions $D$, $\chi, \zeta, \eta$, and $\lambda$ :

$$
\begin{aligned}
& \frac{1}{\rho} \frac{\partial}{\partial \rho}\left(\rho \frac{\partial D}{\partial \rho}\right)+\frac{\partial^{2} D}{\partial z^{2}}-\frac{q^{2} D}{\rho^{2}}-\frac{4 M g\left|\Psi_{0}\right|^{2} D}{\hbar^{2}} \\
& =\frac{2 M\left|\Psi_{0}\right| z}{\hbar^{2}} \partial_{z^{\prime}} V_{t r}\left(\mathbf{r}_{\mathbf{0}}\right), \\
& \frac{1}{\rho} \frac{\partial}{\partial \rho}\left(\rho \frac{\partial \chi}{\partial \rho}\right)+\frac{\partial^{2} \chi}{\partial z^{2}}-\frac{\left(q^{2}+1\right) \chi}{\rho^{2}}-\frac{2 q\left|\Psi_{0}\right| \lambda}{\rho^{2}} \\
& -\frac{4 M g\left|\Psi_{0}\right|^{2} \chi}{\hbar^{2}}=\frac{2 M\left|\Psi_{0}\right|\left|\nabla V_{t r}\right|_{x} \rho}{\hbar^{2}} \\
& -\frac{2 M q\left|\Psi_{0}\right| V_{y}^{\prime}}{\hbar \rho}+\kappa \frac{\partial\left|\Psi_{0}\right|}{\partial \rho}-2 \alpha \frac{\partial^{2}\left|\Psi_{0}\right|}{\partial \rho \partial z}, \\
& \frac{1}{\rho} \frac{\partial}{\partial \rho}\left(\rho \frac{\partial \zeta}{\partial \rho}\right)+\frac{\partial^{2} \zeta}{\partial z^{2}}-\frac{\left(q^{2}+1\right) \zeta}{\rho^{2}}+\frac{2 q\left|\Psi_{0}\right| \eta}{\rho^{2}} \\
& -\frac{4 M g\left|\Psi_{0}\right|^{2} \zeta}{\hbar^{2}}=\frac{2 M\left|\Psi_{0}\right|\left|\nabla V_{t r}\right|_{y} \rho}{\hbar^{2}} \\
& +\frac{2 M q\left|\Psi_{0}\right| V_{x}^{\prime}}{\hbar \rho}-2 \beta \frac{\partial^{2}\left|\Psi_{0}\right|}{\partial \rho \partial z} \\
& \left|\Psi_{0}\right|\left(\frac{\partial^{2}}{\partial \rho^{2}}+\frac{1}{\rho} \frac{\partial}{\partial \rho}+\frac{\partial^{2}}{\partial z^{2}}-\frac{1}{\rho^{2}}\right) \eta \\
& +2\left(\frac{\partial\left|\Psi_{0}\right|}{\partial \rho} \frac{\partial \eta}{\partial \rho}+\frac{\partial\left|\Psi_{0}\right|}{\partial z} \frac{\partial \eta}{\partial z}+\frac{q \zeta}{\rho^{2}}\right) \\
& =\frac{2 M V_{x}^{\prime}}{\hbar} \frac{\partial\left|\Psi_{0}\right|}{\partial \rho}-\frac{2 q \beta}{\rho} \frac{\partial\left|\Psi_{0}\right|}{\partial z}, \\
& \left|\Psi_{0}\right|\left(\frac{\partial^{2}}{\partial \rho^{2}}+\frac{1}{\rho} \frac{\partial}{\partial \rho}+\frac{\partial^{2}}{\partial z^{2}}-\frac{1}{\rho^{2}}\right) \lambda \\
& +2\left(\frac{\partial\left|\Psi_{0}\right|}{\partial \rho} \frac{\partial \lambda}{\partial \rho}+\frac{\partial\left|\Psi_{0}\right|}{\partial z} \frac{\partial \lambda}{\partial z}-\frac{q \chi}{\rho^{2}}\right) \\
& =\frac{2 M V_{y}^{\prime}}{\hbar} \frac{\partial\left|\Psi_{0}\right|}{\partial \rho}+\frac{2 q \alpha}{\rho} \frac{\partial\left|\Psi_{0}\right|}{\partial z}-\frac{q \kappa\left|\Psi_{0}\right|}{\rho} .
\end{aligned}
$$

These equations separate into two connected groups: equations describing perturbations on condensate density [Eqs. (24)-(26)] and equations describing perturbations on the condensate's phase [Eqs. (27) and (28)]. The different groups have different assumptions and transformations that simplify the set of equations.

(i) Substituting $\eta$ and $\lambda$ with $\bar{\eta}+\rho V_{x}^{\prime} M / \hbar$ and $\bar{\lambda}+$ $\rho V_{x}^{\prime} M / \hbar$, removes the $\mathbf{V}^{\prime}$ dependence from the describing equations. As $\rho$ is a solution to $\partial_{\rho}^{2} f(\rho)+\partial_{\rho} f(\rho) / \rho-f(\rho) /$ $\rho^{2}=0$, an arbitrary constant multiplied by $\rho$ can be added to $f(\rho)$ without changing its final solution; consequently, this constant should be chosen to best simplify the equations, removing the $\mathbf{V}^{\prime}$ dependence.

(ii) Derivatives of the phase terms with respect to $z$ can be omitted (and by association $\bar{\eta}$ and $\bar{\lambda}$ ). The gradient of the phase defines the condensate's velocity, with the variation in $z$ therefore inducing flow in $\hat{\mathbf{z}}$. This kind of flow is caused by either the addition of sources and sinks or vortex rings to the BEC. This derivation only considers the behavior of a "straight" vortex in a condensate, and therefore should have no velocity in $\hat{\mathbf{z}}$.

(iii) The phase terms $\partial_{\rho}\left|\Psi_{0}\right| \partial_{\rho} \bar{\eta}$ and $\partial_{\rho}\left|\Psi_{0}\right| \partial_{\rho} \bar{\lambda}$ can be removed. In Eqs. (27) and (28), there are two terms involving the first derivatives of the phase with respect to $\rho$, having 
coefficients $\left|\psi_{0}\right| / \rho$ and $\partial_{\rho}\left|\Psi_{0}\right|$. As mentioned previously, at large $\rho,\left|\Psi_{0}\right|$ is approximately $\left|\Psi_{T F}\right|$, and varies on length scales of the order trap radius $R_{\perp}$; therefore, the change in $\left|\Psi_{0}\right|$ with respect to $\rho$ (varies on $r_{c}$ ) is small compared with $\left|\psi_{0}\right| / \rho$, which is approximately constant, and so has little effect.

(iv) Kinetic-energy terms, from the wave density, can be ignored. In the Thomas-Fermi regime, the kinetic energy from the wave density is assumed to be negligible, giving the wellknown inverted parabola structure $\left(g\left|\Psi_{T F}\right|^{2}=\mu_{T F}-V_{t r}\right)$. As this behavior is expected in the matching region, the Laplacians $\left(\nabla^{2}\right)$ of Eqs. (24)-(26) are approximately 0 , removing the differential behavior from these equations. Applying these assumptions and rearranging Eqs. (24)-(28), we find

$$
\begin{aligned}
& D(\rho, z)=-\frac{z}{2 g\left|\Psi_{0}\right|} \partial_{z^{\prime}} V_{t r}\left(\mathbf{r}_{0}\right), \\
& \chi(\rho, z)=-\frac{\left|\nabla V_{t r}\right|_{x} \rho}{2 g\left|\Psi_{0}\right|}-\frac{\hbar^{2} \kappa}{4 M g\left|\Psi_{0}\right|^{2}} \frac{\partial\left|\Psi_{0}\right|}{\partial \rho} \\
&+\frac{\hbar^{2} \alpha}{2 M g\left|\Psi_{0}\right|^{2}} \frac{\partial^{2}\left|\Psi_{0}\right|}{\partial \rho \partial}-\frac{q \hbar^{2} \bar{\lambda}}{2 M g\left|\Psi_{0}\right| \rho^{2}}, \\
& \zeta(\rho, z)=-\frac{\left|\nabla V_{t r}\right|_{y} \rho}{2 g\left|\Psi_{0}\right|}+\frac{\hbar^{2} \beta}{2 M g\left|\Psi_{0}\right|^{2}} \frac{\partial^{2}\left|\Psi_{0}\right|}{\partial \rho \partial z}+\frac{q \hbar^{2} \bar{\eta}}{2 M g\left|\Psi_{0}\right| \rho^{2}}, \\
&\left|\Psi_{0}\right|\left(\frac{\partial^{2}}{\partial \rho^{2}}+\frac{1}{\rho} \frac{\partial}{\partial \rho}-\frac{1}{\rho^{2}}\right) \bar{\eta}+\frac{2 q \zeta}{\rho^{2}}=-\frac{2 q \beta}{\rho} \frac{\partial\left|\Psi_{0}\right|}{\partial z}, \\
&\left|\Psi_{0}\right|\left(\frac{\partial^{2}}{\partial \rho^{2}}+\frac{1}{\rho} \frac{\partial}{\partial \rho}-\frac{1}{\rho^{2}}\right) \bar{\lambda}-\frac{2 q \chi}{\rho^{2}}=\frac{2 q \alpha}{\rho} \frac{\partial\left|\Psi_{0}\right|}{\partial z}-\frac{q \kappa\left|\Psi_{0}\right|}{\rho}
\end{aligned}
$$

Equation (29) solves for the perturbation $D(\rho, z)$, being the first-order correction to the local Thomas-Fermi profile, i.e., correcting for the changing condensate density in the $z$ direction.

The remaining density perturbations, given by Eqs. (30) and (31), are linear. These equations contain appropriate ThomasFermi, curvature, and $\hat{\mathbf{z}}$ deviation correction terms and a term that depends on a phase perturbation. As a consequence, these will be solved once the phase perturbations are known. Using these to remove $\chi$ and $\zeta$ dependence from Eqs. (32) and (33), two independent differential equations for $\bar{\eta}$ and $\bar{\lambda}$ are found:

$$
\begin{aligned}
& {\left[\frac{\partial^{2}}{\partial \rho^{2}}+\frac{1}{\rho} \frac{\partial}{\partial \rho}-\frac{1}{\rho^{2}}\left(1-\frac{q^{2} \hbar^{2}}{M g\left|\Psi_{0}\right| \rho^{2}}\right)\right] \bar{\eta}} \\
& =-\frac{2 q \beta}{\left|\Psi_{0}\right| \rho} \frac{\partial\left|\Psi_{0}\right|}{\partial z}+\frac{q\left|\nabla V_{t r}\right|_{y}}{g\left|\Psi_{0}\right|^{2} \rho}-\frac{\hbar^{2} q \beta}{M g\left|\Psi_{0}\right|^{3} \rho^{2}} \frac{\partial^{2}\left|\Psi_{0}\right|}{\partial \rho \partial z} \\
& {\left[\frac{\partial^{2}}{\partial \rho^{2}}+\frac{1}{\rho} \frac{\partial}{\partial \rho}-\frac{1}{\rho^{2}}\left(1-\frac{\hbar^{2} q^{2}}{M g\left|\Psi_{0}\right| \rho^{2}}\right)\right] \bar{\lambda}} \\
& =\frac{2 q \alpha}{\left|\Psi_{0}\right| \rho} \frac{\partial\left|\Psi_{0}\right|}{\partial z}-\frac{q\left|\nabla V_{t r}\right|_{x}}{g\left|\Psi_{0}\right|^{2} \rho}-\frac{\hbar^{2} q \kappa}{2 M g\left|\Psi_{0}\right|^{3} \rho^{2}} \frac{\partial\left|\Psi_{0}\right|}{\partial \rho} \\
& \quad+\frac{\hbar^{2} q \alpha}{M g\left|\Psi_{0}\right|^{3} \rho^{2}} \frac{\partial^{2}\left|\Psi_{0}\right|}{\partial \rho \partial z}-\frac{q \kappa}{\rho} .
\end{aligned}
$$

Comparing the relative terms, $\frac{\hbar^{2} q^{2}}{M g\left|\Psi_{0}\right| \rho^{2}}, \frac{\hbar^{2} q \kappa}{2 M g\left|\Psi_{0}\right|^{3} \rho^{2}} \frac{\partial\left|\Psi_{0}\right|}{\partial \rho}$, $\frac{\hbar^{2} q \alpha}{M g\left|\Psi_{0}\right|^{3} \rho^{2}} \frac{\partial^{2}\left|\Psi_{0}\right|}{\partial \rho \partial z}$, and $\frac{\hbar^{2} q \beta}{M g\left|\Psi_{0}\right|^{3} \rho^{2}} \frac{\partial^{2}\left|\Psi_{0}\right|}{\partial \rho \partial z}$ are much smaller than their corresponding counterparts by at least an order of $1 / R_{\perp}$ (especially as $\rho$ gets large). Consequently, these terms do not significantly affect the far-from-core behavior and can be omitted, turning Eqs. (34) and (35) into

$$
\begin{gathered}
\left(\frac{\partial^{2}}{\partial \rho^{2}}+\frac{1}{\rho} \frac{\partial}{\partial \rho}-\frac{1}{\rho^{2}}\right) \bar{\eta} \\
\approx \frac{q \beta}{g\left|\Psi_{T F}\right|^{2} \rho} \frac{\partial V_{t r}}{\partial z}+\frac{2 q\left|\nabla V_{t r}\right|_{y}}{g\left|\Psi_{T F}\right|^{2} \rho}, \\
\left(\frac{\partial^{2}}{\partial \rho^{2}}+\frac{1}{\rho} \frac{\partial}{\partial \rho}-\frac{1}{\rho^{2}}\right) \bar{\lambda} \\
\approx-\frac{q \alpha}{g\left|\Psi_{T F}\right|^{2} \rho} \frac{\partial V_{t r}}{\partial z}-\frac{q \kappa}{\rho}-\frac{q\left|\nabla V_{t r}\right|_{x}}{g\left|\Psi_{T F}\right|^{2} \rho},
\end{gathered}
$$

where $\left|\Psi_{0}\right|$ has been replaced with $\left|\Psi_{T F}\right|$.

Given that $\left|\Psi_{T F}\right|,\left|\nabla V_{t r}\right|$, and $\frac{\partial V_{t r}}{\partial z}$ are constant in the innersolution expansion, these equations are Euler homogeneous equations and have solutions of the form $\rho^{n}$. By solving these equations and making them nondivergent at $\rho=0$, the solutions become

$$
\begin{aligned}
\eta= & \left(\frac{q\left|\nabla V_{t r}\right|_{y}}{2 g\left|\Psi_{T F}\right|^{2}}+\frac{q \beta}{2 g\left|\Psi_{T F}\right|^{2}} \frac{\partial V_{t r}}{\partial z}\right) \rho \ln (\rho) \\
& +\frac{M}{\hbar} \rho V_{x}^{\prime}+\rho A, \\
\lambda=- & \left(\frac{q\left|\nabla V_{t r}\right|_{x}}{2 g\left|\Psi_{T F}\right|^{2}}+\frac{q \alpha}{2 g\left|\Psi_{T F}\right|^{2}} \frac{\partial V_{t r}}{\partial z}+\frac{q \kappa}{2}\right) \rho \ln (\rho) \\
+ & \frac{M}{\hbar} \rho V_{y}^{\prime}+\rho B,
\end{aligned}
$$

where $A$ and $B$ are unknown constants that can depend on $\left|\Psi_{T F}\right|,\left|\nabla V_{t r}\right|, \kappa$, and $\frac{\partial V_{t r}}{\partial z}$.

Therefore, the phase of the inner solution is

$$
\begin{aligned}
S= & q \phi+\left(\frac{q\left|\nabla V_{t r}\right|_{y}}{2 g\left|\Psi_{T F}\right|^{2}}+\frac{q \beta}{2 g\left|\Psi_{T F}\right|^{2}} \frac{\partial V_{t r}}{\partial z}\right) x \ln (\rho) \\
& -\left(\frac{q\left|\nabla V_{t r}\right|_{x}}{2 g\left|\Psi_{T F}\right|^{2}}+\frac{q \alpha}{2 g\left|\Psi_{T F}\right|^{2}} \frac{\partial V_{t r}}{\partial z}+\frac{q \kappa}{2}\right) y \ln (\rho) \\
& +x A+y B+\frac{M}{\hbar} x V_{x}^{\prime}+\frac{M}{\hbar} y V_{y}^{\prime}
\end{aligned}
$$

or

$$
\begin{aligned}
S= & q \phi+\mathbf{r} \cdot \frac{M}{\hbar}\left(\mathbf{V}+\mathbf{\Omega} \times \mathbf{r}_{\mathbf{0}}\right) \\
& -\mathbf{r} \cdot \frac{q \ln (\rho)}{2 g\left|\Psi_{T F}\right|^{2}}\left[\kappa g\left|\Psi_{T F}\right|^{2} \hat{\mathbf{b}}+\hat{\mathbf{z}} \times \nabla V_{t r}\left(\mathbf{r}_{\mathbf{0}}\right)\right] \\
& +\mathbf{r} \cdot \frac{q \ln (\rho)}{2 g\left|\Psi_{T F}\right|^{2}}\left[\left.(\hat{\mathbf{z}} \times \hat{\mathbf{t}}) \frac{\partial V_{t r}}{\partial z}\right|_{\mathbf{r}=\mathbf{r}_{0}}\right] \\
& +\mathbf{r} \cdot \mathbf{E}\left[\kappa \hat{\mathbf{b}}, \frac{\hat{\mathbf{z}} \times \nabla V_{t r}\left(\mathbf{r}_{\mathbf{0}}\right)}{g\left|\Psi_{T F}\right|^{2}},\left.\frac{\hat{\mathbf{z}} \times \hat{\mathbf{t}}}{g\left|\Psi_{T F}\right|^{2}} \frac{\partial V_{t r}}{\partial z}\right|_{\mathbf{r}=\mathbf{r}_{\mathbf{0}}}, \mathbf{r}_{\mathbf{0}}\right],
\end{aligned}
$$

as $\hat{\mathbf{t}}=\{\alpha, \beta, 1\}$, and $\mathbf{E}=\{A, B, 0\}$. The dependent terms in $\mathbf{E}$ are written down here to emphasize that the constant can depend on $\left|\Psi_{T F}\right|,\left|\nabla V_{t r}\right|, \kappa$, and $\frac{\partial V_{t r}}{\partial z}$, with the structure of the 
dependents chosen because of the structure of the other terms in Eq. (41).

Using Eqs. (38) and (39), the density perturbations are

$$
\begin{aligned}
D= & -\frac{z}{2 g\left|\Psi_{T F}\right|} \partial_{z^{\prime}} V_{t r}\left(\mathbf{r}_{\mathbf{0}}\right), \\
\chi= & -\frac{\left|\nabla V_{t r}\right|_{x} \rho}{2 g\left|\Psi_{T F}\right|}-\frac{\hbar^{2} \kappa}{4 M g\left|\Psi_{T F}\right|^{2}} \frac{\partial\left|\Psi_{T F}\right|}{\partial \rho} \\
& +\frac{\hbar^{2} q^{2}\left(\left|\nabla V_{t r}\right|_{x}+\alpha \frac{\partial V_{t r}}{\partial z}+\kappa g\left|\Psi_{T F}\right|^{2}\right) \ln (\rho)}{4 M g^{2}\left|\Psi_{T F}\right|^{3} \rho} \\
& +\frac{\hbar^{2} \alpha}{2 M g\left|\Psi_{T F}\right|^{2}} \frac{\partial^{2}\left|\Psi_{T F}\right|}{\partial \rho \partial z}-\frac{q \hbar^{2} B}{2 M g\left|\Psi_{T F}\right| \rho}, \\
\zeta= & -\frac{\left|\nabla V_{t r}\right|_{y} \rho}{2 g\left|\Psi_{T F}\right|}+\frac{\hbar^{2} \beta}{2 M g\left|\Psi_{T F}\right|^{2}} \frac{\partial^{2}\left|\Psi_{T F}\right|}{\partial \rho \partial z} \\
& +\frac{q^{2} \hbar^{2}\left(\left|\nabla V_{t r}\right|_{y}+\beta \frac{\partial V_{t r}}{\partial z}\right) \ln (\rho)}{4 M g^{2}\left|\Psi_{T F}\right|^{3} \rho}+\frac{q \hbar^{2} A}{2 M g\left|\Psi_{T F}\right| \rho} .
\end{aligned}
$$

These density perturbations are independent of the vortex core's velocity $(\mathbf{V})$, being, to the lowest order, corrections to the local Thomas-Fermi profile. Hence these solutions already match the Thomas-Fermi density profile of the outer region. This indicates that the perturbations on a vortex line predominantly affect the inner phase of the condensate [Eq. (41)], which through matching to the outer-regions phase should give the desired vortex relation.

\section{THE PHASE OF THE OUTER REGION}

Far from the vortex core, the BEC's wave-function density has the Thomas-Fermi profile, and its phase $(S)$ solves

$$
\begin{gathered}
\nabla \cdot\left(\left|\Psi_{T F}\right|^{2} \nabla S\right)-\frac{M}{\hbar} \boldsymbol{\Omega} \cdot(\mathbf{r} \times \nabla)\left|\Psi_{T F}\right|^{2}=0, \\
\hat{\mathbf{z}} \cdot \nabla \times(\nabla S)=2 \pi q \delta^{(2)}\left(\boldsymbol{\rho}-\boldsymbol{\rho}_{\mathbf{0}}\right),
\end{gathered}
$$

where $\delta^{(2)}\left(\boldsymbol{\rho}-\boldsymbol{\rho}_{\mathbf{0}}\right)$ is the delta function in $\rho$ and $\phi$.

These equations are linear, and therefore allow $S$ to be solved in two separate components: one dealing with rotation [the particular solution of Eq. (45), $S_{\Omega}$ ] and one with everything else $\left(S_{c}\right)$ :

$$
\begin{gathered}
\nabla \cdot\left(\left|\Psi_{T F}\right|^{2} \nabla S_{\Omega}\right)-\frac{M}{\hbar} \boldsymbol{\Omega} \cdot(\mathbf{r} \times \nabla)\left|\Psi_{T F}\right|^{2}=0, \\
\nabla \times\left(\nabla S_{\Omega}\right)=0, \\
\nabla \cdot\left(\left|\Psi_{T F}\right|^{2} \nabla S_{c}\right)=0, \\
\hat{\mathbf{z}} \cdot \nabla \times\left(\nabla S_{c}\right)=2 \pi q \delta^{(2)}\left(\rho-\rho_{0}\right) .
\end{gathered}
$$

Assuming $\boldsymbol{\Omega}=\Omega \hat{\mathbf{z}}$, Eqs. (47) and (48) have the solution

$$
S_{\Omega}=-\frac{M}{\hbar} \frac{\left(\omega_{x}^{2}-\omega_{y}^{2}\right)}{\left(\omega_{x}^{2}+\omega_{y}^{2}\right)} \Omega x y,
$$

where $\omega_{x}$ and $\omega_{y}$ are the trapping frequencies in $x$ and $y$, respectively.

Transforming into the local coordinate system of the line $\left(\mathbf{r} \rightarrow \mathbf{r}+\mathbf{r}_{\mathbf{0}}\right)$, Eq. (51) becomes

$$
S_{\Omega} \rightarrow \frac{M}{\hbar}\left[\left(\boldsymbol{\Omega} \times \mathbf{r}_{\mathbf{0}}\right)+2 \frac{\nabla V_{t r}\left(\mathbf{r}_{\mathbf{0}}\right) \times \boldsymbol{\Omega}}{\nabla_{\perp}^{2} V_{t r}\left(\mathbf{r}_{\mathbf{0}}\right)}\right] \cdot \mathbf{r} .
$$

$S_{c}$ is not as simple to solve, with no exact solution existing for arbitrary $\boldsymbol{\rho}_{\mathbf{0}}$. The gradient of $S_{c}$, however, can be determined and consequently compared with its innersolution equivalent. To do this, $\nabla S_{c}$ is written as $-q[\nabla \times$ $\left.\left(\left|\Psi_{T F}\right|^{2} \Phi \hat{\mathbf{z}}\right)\right] /\left|\Psi_{T F}\right|^{2}$, so that it automatically satisfies Eq. (49), and turns Eq. (50) into

$$
\begin{aligned}
\hat{\mathbf{z}} \cdot \nabla \times\left(\nabla S_{c}\right) & =\nabla \cdot\left(\nabla S_{c} \times \hat{\mathbf{z}}\right) \\
& =\nabla \cdot\left[\nabla_{\perp} \Phi+\Phi \nabla_{\perp} \ln \left(\left|\Psi_{T F}\right|^{2}\right)\right] \\
& =2 \pi \delta^{(2)}\left(\rho-\rho_{0}\right),
\end{aligned}
$$

which through rearrangement becomes

$$
\begin{aligned}
& e^{-\ln \left|\Psi_{T F}\right|} \nabla_{\perp}^{2}\left(\Phi e^{\ln \left|\Psi_{T F}\right|}\right)-\nabla_{\perp}^{2}\left(e^{-\ln \left|\Psi_{T F}\right|}\right) \Phi e^{\ln \left|\Psi_{T F}\right|} \\
& \quad=2 \pi \delta^{(2)}\left(\rho-\rho_{\mathbf{0}}\right) .
\end{aligned}
$$

By transforming Eq. (54) into the local coordinates of the vortex line, and taking $\nabla_{\perp}^{2} e^{-\ln \left|\Psi_{T F}\right|}$ to be approximately $e^{-\ln \left|\Psi_{T F}\right|} \nabla_{\perp}^{2} V_{t r} / 2 g\left|\Psi_{T F}\right|^{2}$, it becomes

$$
\nabla_{\perp}^{2}\left(\Phi e^{\ln \left|\Psi_{T F}\right|}\right)-\frac{\nabla_{\perp}^{2} V_{t r}}{2 g\left|\Psi_{T F}\right|^{2}} \Phi e^{\ln \left|\Psi_{T F}\right|}=2 \pi \delta^{(2)}(\rho) e^{\ln \left|\Psi_{T F}\right|} .
$$

In this outer region, there should be no flow in the $\hat{\rho}$ or $\hat{\mathbf{z}}$, and therefore $\Phi$ is purely a function of $\rho$. Furthermore, Anglin [28] showed that in a complete hydrodynamic calculation, flow only arises from the vortex's vorticity, thereby indicating that the homogeneous solution to Eq. (55) is irrelevant. Hence the solution to Eq. (55) is

$$
\Phi=-K_{0}\left(\rho \sqrt{\frac{\nabla_{\perp}^{2} V_{t r}}{2 g\left|\Psi_{T F}\right|^{2}}}\right)
$$

where $K_{0}$ is a modified Bessel function of the second kind, and $V_{t r}$ and $g\left|\Psi_{T F}\right|^{2}$ are implicitly functions of $\mathbf{r}_{\mathbf{0}}$.

For small $\rho$,

$$
\Phi \approx \ln \left(\frac{e^{c} \rho}{2 R_{\perp}}\right)
$$

where $c=0.577 \ldots$ is the Euler constant, and $\nabla_{\perp}^{2} V_{t r} /$ $2 g\left|\Psi_{T F}\right|^{2}$ has been approximated by $1 / R_{\perp}$ (as $\nabla_{\perp}^{2} V_{t r} \approx M \omega_{\perp}^{2}$ and $\left.g\left|\Psi_{T F}\right|^{2} \approx \mu_{T F}=\frac{1}{2} M \omega_{\perp}^{2} R_{\perp}^{2}\right)$.

Using this, we get

$$
\begin{aligned}
\nabla S_{c}= & \frac{-q}{\left|\Psi_{T F}\right|^{2}}\left[\nabla \times\left|\Psi_{T F}\right|^{2} \ln \left(\frac{e^{c} \rho}{2 R_{\perp}}\right) \hat{\mathbf{z}}\right] \\
= & -q \nabla \times \ln \left(\frac{e^{c} \rho}{2 R_{\perp}}\right) \hat{\mathbf{z}} \\
& -q \ln \left(\frac{e^{c} \rho}{2 R_{\perp}}\right) \nabla \times \ln \left|\Psi_{T F}\right|^{2} \hat{\mathbf{z}} \\
= & \frac{q}{\rho} \hat{\phi}-\frac{q \ln \left(\frac{e^{c} \rho}{2 R_{\perp}}\right)}{g\left|\Psi_{T F}\right|^{2}} \hat{\mathbf{z}} \times \nabla V_{t r}\left(\mathbf{r}_{\mathbf{0}}\right),
\end{aligned}
$$

having used the identity $\nabla \times A(\mathbf{r}) \hat{\mathbf{z}}=-\hat{\mathbf{z}} \times \nabla A(\mathbf{r})$.

Therefore, the outer phase is fully described by Eqs. (58) and (52). Through matching these equations with the inner phase [Eq. (41)], a relationship for the velocity of the vortex line is determined. 


\section{MATCHING THE INNER AND OUTER SOLUTIONS}

To match the inner and outer solutions, the inner phase must be divided into the same $S_{\Omega}$ and $S_{c}$ structure as the outer solution. By assuming that the core velocity $\mathbf{V}$ can be written as a rotational $\mathbf{V}_{\boldsymbol{\Omega}}$ and other $\mathbf{V}_{\mathbf{c}}$ component, Eq. (41) separates into

$$
S_{\Omega_{i}}=\frac{M}{\hbar}\left(\mathbf{V}_{\boldsymbol{\Omega}}+\mathbf{\Omega} \times \mathbf{r}_{\mathbf{0}}\right) \cdot \mathbf{r}
$$

and

$$
\begin{aligned}
S_{c_{i}}= & q \phi+\mathbf{r} \cdot \frac{M}{\hbar} \mathbf{V}_{\mathbf{c}}-\mathbf{r} \cdot \frac{q \ln (\rho)}{2} \kappa \hat{\mathbf{b}} \\
& -\mathbf{r} \cdot \frac{q \ln (\rho)}{2 g\left|\Psi_{T F}\right|^{2}}\left[\hat{\mathbf{z}} \times \nabla V_{t r}\left(\mathbf{r}_{\mathbf{0}}\right)+\left.(\hat{\mathbf{z}} \times \hat{\mathbf{t}}) \frac{\partial V_{t r}}{\partial z}\right|_{\mathbf{r}=\mathbf{r}_{0}}\right] \\
& +\mathbf{r} \cdot \mathbf{E}\left[\kappa \hat{\mathbf{b}}, \frac{\hat{\mathbf{z}} \times \nabla V_{t r}\left(\mathbf{r}_{\mathbf{0}}\right)}{g\left|\Psi_{T F}\right|^{2}},\left.\frac{\hat{\mathbf{z}} \times \hat{\mathbf{t}}}{g\left|\Psi_{T F}\right|^{2}} \frac{\partial V_{t r}}{\partial z}\right|_{\mathbf{r}=\mathbf{r}_{\mathbf{0}}}, \mathbf{r}_{\mathbf{0}}\right]
\end{aligned}
$$

which can be compared to the outer $S_{\Omega}$ [Eq. (52)] and $S_{c}$ [Eq. (58)] to find the matching condition for the velocity of the line.

A comparison of Eq. (59) with Eq. (52) shows that $\mathbf{V}_{\boldsymbol{\Omega}}$ is

$$
\mathbf{V}_{\boldsymbol{\Omega}}\left(\mathbf{r}_{\mathbf{0}}\right)=2 \frac{\nabla V_{t r}\left(\mathbf{r}_{\mathbf{0}}\right) \times \boldsymbol{\Omega}}{\nabla_{\perp}^{2} V_{t r}\left(\mathbf{r}_{\mathbf{0}}\right)}
$$

To determine $\mathbf{V}_{\mathbf{c}}$, however, $\nabla S_{c}$ of the two regions must be matched. Using Eq. (60), $\nabla S_{c_{i}}$ for large $\rho$ is

$$
\begin{aligned}
\nabla S_{c_{i}}= & \frac{q}{\rho} \hat{\phi}+\frac{M}{\hbar} \mathbf{V}_{\mathbf{c}}\left(\mathbf{r}_{\mathbf{0}}\right)-\frac{q \kappa \hat{\mathbf{b}}}{2}[\ln (\rho)+\hat{\rho} \otimes \hat{\rho}] \\
& -\frac{q \hat{\mathbf{z}} \times \nabla V_{t r}\left(\mathbf{r}_{\mathbf{0}}\right)}{2 g\left|\Psi_{T F}\right|^{2}}[\ln (\rho)+\hat{\rho} \otimes \hat{\rho}] \\
& -\frac{\left.q(\hat{\mathbf{z}} \times \hat{\mathbf{t}}) \frac{\partial V_{t r}}{\partial z}\right|_{\mathbf{r}=\mathbf{r}_{0}}[\ln (\rho)+\hat{\rho} \otimes \hat{\rho}]+\mathbf{E},}{2 g\left|\Psi_{T F}\right|^{2}}[
\end{aligned}
$$

where for large $\rho, \frac{\mathbf{r}}{\rho} \approx \hat{\rho}$ and $\nabla \mathbf{r}=\mathbf{I}$ (where $\mathbf{I}$ is the identity matrix).

By matching Eqs. (62) and (58), at the vortex core radius $r_{c}$, the equation for $\mathbf{V}_{\mathbf{c}}$ becomes

$$
\begin{aligned}
\mathbf{V}_{\mathbf{c}}\left(\mathbf{r}_{\mathbf{0}}\right)= & \frac{\hbar q \kappa \hat{\mathbf{b}}}{2 M}\left[\ln \left(r_{c}\right)+\hat{\rho} \otimes \hat{\rho}\right] \\
& +\frac{\hbar q \hat{\mathbf{z}} \times \nabla V_{t r}\left(\mathbf{r}_{\mathbf{0}}\right)}{2 M g\left|\Psi_{T F}\right|^{2}}\left[\ln \left(r_{c}\right)+\hat{\rho} \otimes \hat{\rho}\right] \\
& +\frac{\left.\hbar q(\hat{\mathbf{z}} \times \hat{\mathbf{t}}) \frac{\partial V_{t r}}{\partial z}\right|_{\mathbf{r}=\mathbf{r}_{0}}\left[\ln \left(r_{c}\right)+\hat{\rho} \otimes \hat{\rho}\right]}{2 M g\left|\Psi_{T F}\right|^{2}} \\
& +\mathbf{E}^{\prime}\left[\kappa \hat{\mathbf{b}}, \frac{\hat{\mathbf{z}} \times \nabla V_{t r}\left(\mathbf{r}_{\mathbf{0}}\right)}{g\left|\Psi_{T F}\right|^{2}},\left.\frac{\hat{\mathbf{z}} \times \hat{\mathbf{t}}}{g\left|\Psi_{T F}\right|^{2}} \frac{\partial V_{t r}}{\partial z}\right|_{\mathbf{r}=\mathbf{r}_{\mathbf{0}}}, \mathbf{r}_{\mathbf{0}}\right] \\
& -\frac{\hbar q \ln \left(\frac{e^{c} r_{c}}{2 R_{\perp}}\right)}{M g\left|\Psi_{T F}\right|^{2}}\left[\hat{\mathbf{z}} \times \nabla V_{t r}\left(\mathbf{r}_{\mathbf{0}}\right)\right],
\end{aligned}
$$

where $\mathbf{E}^{\prime}=-\hbar \mathbf{E} / M$.
Combining $\mathbf{V}_{\mathbf{c}}$ [Eq. (63)] and $\mathbf{V}_{\boldsymbol{\Omega}}$ [Eq. (61)], the relation for velocity of the vortex line is

$$
\begin{aligned}
\mathbf{V}\left(\mathbf{r}_{\mathbf{0}}\right)= & \frac{\hbar q \kappa \hat{\mathbf{b}}}{2 M}\left[\ln \left(r_{c}\right)+\hat{\rho} \otimes \hat{\rho}\right] \\
& +\frac{\hbar q \hat{\mathbf{z}} \times \nabla V_{t r}\left(\mathbf{r}_{\mathbf{0}}\right)}{2 M g\left|\Psi_{T F}\right|^{2}}\left[\ln \left(r_{c}\right)+\hat{\rho} \otimes \hat{\rho}\right] \\
& +\frac{\left.\hbar q(\hat{\mathbf{z}} \times \hat{\mathbf{t}}) \frac{\partial V_{t r}}{\partial z}\right|_{\mathbf{r}=\mathbf{r}_{\mathbf{0}}}}{2 M g\left|\Psi_{T F}\right|^{2}}\left[\ln \left(r_{c}\right)+\hat{\rho} \otimes \hat{\rho}\right] \\
& +\mathbf{E}^{\prime}\left[\kappa \hat{\mathbf{b}}, \frac{\hat{\mathbf{z}} \times \nabla V_{t r}\left(\mathbf{r}_{\mathbf{0}}\right)}{g\left|\Psi_{T F}\right|^{2}},\left.\frac{\hat{\mathbf{z}} \times \hat{\mathbf{t}}}{g\left|\Psi_{T F}\right|^{2}} \frac{\partial V_{t r}}{\partial z}\right|_{\mathbf{r}=\mathbf{r}_{\mathbf{0}}}, \mathbf{r}_{\mathbf{0}}\right] \\
& -\frac{\hbar q \ln \left(\frac{e^{c} r_{c}}{2 R_{\perp}}\right)}{M g\left|\Psi_{T F}\right|^{2}}\left[\hat{\mathbf{z}} \times \nabla V_{t r}\left(\mathbf{r}_{\mathbf{0}}\right)\right]+2 \frac{\nabla V_{t r}\left(\mathbf{r}_{\mathbf{0}}\right) \times \mathbf{\Omega}}{\nabla_{\perp}^{2} V_{t r}\left(\mathbf{r}_{\mathbf{0}}\right)},
\end{aligned}
$$

where $\hat{\rho}$ is effectively the $\rho$ vector in the condensate coordinates (the difference between $\hat{\rho}$ of the vortex line and $\hat{\rho}$ of the condensate is negligible at large distances).

This equation indicates how the local velocity of the vortex line behaves in response to the line's position and curvature, and therefore reveals the local behavior of a vortex line. Furthermore, the $\hat{\mathbf{z}}$ dependence in this equation indicates that it is coordinate dependent. This is because the initially perturbed system has a natural direction, which is the alignment of the straight centered vortex. The $\hat{\mathbf{z}}$ dependence in Eq. (64) thereby reflects this initial vortex line orientation.

Equation (64) also transforms to give a system of differential equations that describes the complete vortex behavior. As the vortex line is a one-to-one function in $z$, the vortex line's shape and motion are fully parametrized through two functions, $\rho(t, z)$ and $\phi(t, z)$. This allows any point on the line to be represented by $\mathbf{r}_{\mathbf{0}}=\rho(t, z) \hat{\rho}+\phi(t, z) \hat{\phi}+z \hat{\mathbf{z}}$, with velocity and tangent vectors, respectively,

$$
\begin{aligned}
\mathbf{V} & =\frac{\partial \rho}{\partial t} \hat{\rho}+\rho \frac{\partial \phi}{\partial t} \hat{\phi}, \\
\hat{\mathbf{t}} & =\frac{\partial \rho}{\partial z} \hat{\rho}+\rho \frac{\partial \phi}{\partial z} \hat{\phi}+\hat{\mathbf{z}},
\end{aligned}
$$

and $\kappa \hat{\mathbf{b}}\left(\approx \hat{\mathbf{z}} \times \mathbf{k}\right.$, where $\left.\mathbf{k}=\partial_{s}^{2} \mathbf{r}_{\mathbf{0}}\right)$ as

$$
\kappa \hat{\mathbf{b}}=-\left(\rho \frac{\partial^{2} \phi}{\partial z^{2}}+2 \frac{\partial \rho}{\partial z} \frac{\partial \phi}{\partial z}\right) \hat{\rho}+\left[\frac{\partial^{2} \rho}{\partial z^{2}}-\rho\left(\frac{\partial \phi}{\partial z}\right)^{2}\right] \hat{\phi}
$$

where $\rho$ and $\phi$ are implicitly functions of $t$ and $z$, and $z$ has been approximated to be the arc length of the curve.

As a result, the differential equations describing the full vortex's shape and motion are

$$
\begin{aligned}
\frac{\partial \rho}{\partial t}= & -\frac{\hbar q\left[\ln \left(r_{c}\right)+1\right]}{2 M}\left(\rho \frac{\partial^{2} \phi}{\partial z^{2}}+2 \frac{\partial \rho}{\partial z} \frac{\partial \phi}{\partial z}\right) \\
& -\frac{\hbar q\left[\ln \left(r_{c}\right)+1\right]}{2 M g\left|\Psi_{T F}\right|^{2}}\left(\frac{1}{\rho} \frac{\partial V_{t r}}{\partial \phi}+\rho \frac{\partial \phi}{\partial z} \frac{\partial V_{t r}}{\partial z}\right) \\
& +\frac{2 \Omega}{\rho \nabla_{\perp}^{2} V_{t r}} \frac{\partial V_{t r}}{\partial \phi}+\frac{\hbar q \ln \left(\frac{e^{c} r_{c}}{2 R_{\perp}}\right)}{\rho M g\left|\Psi_{T F}\right|^{2}} \frac{\partial V_{t r}}{\partial \phi}+\hat{\rho} \cdot \mathbf{E}^{\prime},
\end{aligned}
$$




$$
\begin{aligned}
\rho \frac{\partial \phi}{\partial t}= & \frac{\hbar q \ln \left(r_{c}\right)}{2 M}\left[\frac{\partial^{2} \rho}{\partial z^{2}}-\rho\left(\frac{\partial \phi}{\partial z}\right)^{2}\right] \\
& +\frac{\hbar q \ln \left(r_{c}\right)}{2 M g\left|\Psi_{T F}\right|^{2}}\left(\frac{\partial V_{t r}}{\partial \rho}+\frac{\partial \rho}{\partial z} \frac{\partial V_{t r}}{\partial z}\right) \\
& -\frac{2 \Omega}{\nabla_{\perp}^{2} V_{t r}} \frac{\partial V_{t r}}{\partial \rho}-\frac{\hbar q \ln \left(\frac{e^{c} r_{c}}{2 R_{\perp}}\right)}{M g\left|\Psi_{T F}\right|^{2}} \frac{\partial V_{t r}}{\partial \rho}+\hat{\phi} \cdot \mathbf{E}^{\prime} .
\end{aligned}
$$

The solutions of Eqs. (68) and (69) describe a vortex's shape and motion, and show the steady-state structures and waves that the vortex supports. However, before these structures are determined, the unknown constant $\mathbf{E}^{\prime}$ needs to be found.

\section{DETERMINATION OF E'}

Before investigating Eqs. (68) and (69), $\mathbf{E}^{\prime}$ needs to be determined. This can be done through comparing the specific results from these equations to previously determined physical scenarios.

Assuming that the trapping and curving dependence in $\mathbf{E}^{\prime}$ can be treated separately, two different scenarios need to be considered: one indicating a straight vortex's reaction to the trap confinement and one describing behavior of wave perturbations on the line in the absence of confinement.

In a pancake-shaped condensate, it has been shown that the vortex lines are straight, while for more-cigar-shaped traps, the line tends to bend at the edges [15]. Such straight lines are easy to work with mathematically and so the energetics and behavior of these lines have been investigated [12,14]. These investigations revealed that an off-centered straight-line vortex precesses around a cylindrical condensate in the direction of the vortex's rotation, with a precession frequency

$$
\frac{\partial \phi}{\partial t}=\frac{3 \hbar \ln \left(\frac{R_{\perp}}{r_{c}}\right)}{2 M R_{\perp}^{2}\left(1-\frac{\rho^{2}}{R_{\perp}^{2}}\right)}-\Omega .
$$

Applying similar conditions to Eqs. (68) and (69) (discarding $z$ derivatives and setting $\left.V_{t r}=M \omega_{\perp}^{2} \rho^{2} / 2+M \omega_{z}^{2} z^{2} / 2\right)$ and setting $g\left|\Psi_{T F}\right|^{2} \approx \frac{1}{2} M \omega_{\perp}^{2} R_{\perp}^{2}\left(1-\frac{\rho^{2}}{R_{\perp}^{2}}\right)$, the describing equations become

$$
\begin{gathered}
\frac{\partial \rho}{\partial t}=\hat{\rho} \cdot \mathbf{E}^{\prime}\left[0, \frac{2 \rho \hat{\phi}}{R_{\perp}^{2}\left(1-\frac{\rho^{2}}{R_{\perp}^{2}}\right)}, 0, \mathbf{r}\right], \\
\rho \frac{\partial \phi}{\partial t}=-\frac{\hbar \ln \left(\frac{e^{2 c} r_{c}}{4 R_{\perp}^{2}}\right) \rho}{M R_{\perp}^{2}\left(1-\frac{\rho^{2}}{R_{\perp}^{2}}\right)}-\Omega \rho+\hat{\phi} \cdot \mathbf{E}^{\prime}\left[0, \frac{2 \rho \hat{\phi}}{R_{\perp}^{2}\left(1-\frac{\rho^{2}}{R_{\perp}^{2}}\right)}, 0, \mathbf{r}\right] .
\end{gathered}
$$

When matched to Eq. (70), these equations become

$$
\hat{\rho} \cdot \mathbf{E}^{\prime}\left[0, \frac{2 \rho \hat{\phi}}{R_{\perp}^{2}\left(1-\frac{\rho^{2}}{R_{\perp}^{2}}\right)}, 0, \mathbf{r}\right]=0,
$$

$$
\hat{\phi} \cdot \mathbf{E}^{\prime}\left[0, \frac{2 \rho \hat{\phi}}{R_{\perp}^{2}\left(1-\frac{\rho^{2}}{R_{\perp}^{2}}\right)}, 0, \mathbf{r}\right]=\frac{\hbar \ln \left(\frac{e^{2 c}}{4 \sqrt{R_{\perp} r_{c}}}\right) \rho}{M R_{\perp}^{2}\left(1-\frac{\rho^{2}}{R_{\perp}^{2}}\right)} .
$$

This suggests that the trap dependence in the constant term has the form

$$
\begin{aligned}
& \mathbf{E}^{\prime}\left(\kappa \hat{\mathbf{b}}, \frac{\hat{\mathbf{z}} \times \nabla V_{t r}}{g\left|\Psi_{T F}\right|^{2}}, \frac{\hat{\mathbf{z}} \times \hat{\mathbf{t}}}{g\left|\Psi_{T F}\right|^{2}} \frac{\partial V_{t r}}{\partial z}, \mathbf{r}\right) \\
& =\frac{\hbar q \ln \left(\frac{e^{2 c}}{4 \sqrt{R_{\perp} r_{c}}}\right)\left(\hat{\mathbf{z}} \times \nabla V_{t r}\right)}{2 M g\left|\Psi_{T F}\right|^{2}}+\mathbf{F}(\kappa \hat{\mathbf{b}}, \mathbf{r}),
\end{aligned}
$$

where $\mathbf{F}$ is an unknown constant that describes the curvature behavior.

Note that this condition, though intending to contain all trap dependence, does not contain information on the line's reaction to the $z$ trapping of the condensate. This indicates that another condition relating specifically to this term is necessary (visible through the $\ln$ term). Unfortunately, a suitable comparison was not found and therefore this term could not be calibrated.

To determine the curvature-related behavior of the constant, the behavior of a perturbed vortex (in a untrapped condensate) needs to be known. It has been shown, theoretically, that vortices in BECs support helical-wave perturbations [10] and therefore are an ideal example. These waves rotate in the opposite direction to the rotation of the vortex, and in a uniform condensate have a dispersion relation (for large wavelengths) of

$$
\omega=\frac{\hbar k^{2}}{2 M} \ln \left(\frac{1}{|k| r_{c}}\right)
$$

where $\omega$ is the wave frequency, $k$ is the wave number, and $r_{c}$ is the vortex core radius. Hence by comparing this dispersion relation to the equivalent derived from Eqs. (68) and (69), the form of $\mathbf{F}$ can be determined.

A helical wave generally has the form $x=a \sin (k z-\omega t+$ $\left.\phi_{0}\right)$ and $y=a \cos \left(k z-\omega t+\phi_{0}\right)$, which when transformed into cylindrical coordinates is $\rho=a$ and $\phi=k z-\omega t+\phi_{0}$ (with $a$ being the wave amplitude and $\phi_{0}$ being an arbitrary phase constant). By substituting this parametrization into Eqs. (68) and (69), they become

$$
0=-\frac{\hbar\left[\ln \left(r_{c}\right)+1\right]}{2 M g\left|\Psi_{T F}\right|^{2}}(0)+\hat{\rho} \cdot \mathbf{F}\left(-a k^{2} \hat{\phi}, \mathbf{r}\right)
$$

and

$$
-a \omega=-\frac{\hbar a k^{2} \ln \left(r_{c}\right)}{2 M}+\hat{\phi} \cdot \mathbf{F}\left(-a k^{2} \hat{\phi}, \mathbf{r}\right)
$$

Hence, the conditions $\mathbf{F}$ satisfies are

$$
\begin{gathered}
\hat{\rho} \cdot \mathbf{F}\left(-a k^{2} \hat{\phi}, \mathbf{r}\right)=0, \\
\hat{\phi} \cdot \mathbf{F}\left(-a k^{2} \hat{\phi}, \mathbf{r}\right)=a \frac{\hbar k^{2}}{2 M} \ln \left(|k| r_{c}^{2}\right) .
\end{gathered}
$$

Since all of the wave-number dependence in $\mathbf{F}$ must come from the $\kappa \hat{\mathbf{b}}$ term, a suitable form becomes

$$
\mathbf{F}=\frac{\hbar q \ln \left(\frac{1}{r_{c}^{2}} \sqrt{\frac{-\rho}{\kappa \hat{\mathbf{b}} \cdot \hat{\phi}}}\right)}{2 M} \kappa \hat{\mathbf{b}}
$$


By substituting the full form of $\mathbf{E}^{\prime}$ into Eq. (64), the velocity condition becomes

$$
\begin{aligned}
\mathbf{V}\left(\mathbf{r}_{\mathbf{0}}\right)= & \frac{\left.\hbar q(\hat{\mathbf{z}} \times \hat{\mathbf{t}}) \frac{\partial V_{t r}}{\partial z}\right|_{\mathbf{r}=\mathbf{r}_{\mathbf{0}}}}{2 M g\left|\Psi_{T F}\right|^{2}}\left[\ln \left(r_{c}\right)+\hat{\rho} \otimes \hat{\rho}\right] \\
& +\frac{\hbar q \kappa \hat{\mathbf{b}}}{2 M}\left[\ln \left(\frac{1}{r_{c}} \sqrt{\frac{-\rho_{0}}{\kappa \hat{\mathbf{b}} \cdot \hat{\phi}}}\right)+\hat{\rho} \otimes \hat{\rho}\right] \\
& +\frac{\hbar q\left[\hat{\mathbf{z}} \times \nabla V_{t r}\left(\mathbf{r}_{\mathbf{0}}\right)\right]}{2 M g\left|\Psi_{T F}\right|^{2}}\left[\frac{3}{2} \ln \left(\frac{R_{\perp}}{r_{c}}\right)+\hat{\rho} \otimes \hat{\rho}\right] \\
& +2 \frac{\nabla V_{t r}\left(\mathbf{r}_{\mathbf{0}}\right) \times \boldsymbol{\Omega}}{\nabla_{\perp}^{2} V_{t r}\left(\mathbf{r}_{\mathbf{0}}\right)} .
\end{aligned}
$$

This is an almost complete relation for the velocity of the vortex line, missing the calibration of the $\partial_{z} V_{t r}$ term [as evidenced by the units in the multiplying $\left.\operatorname{logarithm} \ln \left(r_{c}\right)\right]$. As a suitable analytic form for this comparison could not be found, and it does not affect any of the latter calculations performed, this issue will be left for future work.

When transformed into a differential form (as per Sec. VI), this equation gives

$$
\begin{aligned}
\frac{\partial \rho}{\partial t}= & -\frac{\hbar q \rho\left[\ln \left(r_{c}\right)+1\right]}{2 M g\left|\Psi_{T F}\right|^{2}} \frac{\partial \phi}{\partial z} \frac{\partial V_{t r}}{\partial z} \\
& -\frac{\hbar q\left\{\ln \left[\frac{1}{r_{c}} \sqrt{\frac{\rho}{\rho\left(\partial_{z} \phi\right)^{2}-\partial_{z}^{2} \rho}}\right]+1\right\}}{2 M}\left(\rho \frac{\partial^{2} \phi}{\partial z^{2}}+2 \frac{\partial \rho}{\partial z} \frac{\partial \phi}{\partial z}\right) \\
& -\frac{3 \hbar q\left[\ln \left(\frac{R_{\perp}}{r_{c}}\right)+\frac{2}{3}\right]}{4 \rho M g\left|\Psi_{T F}\right|^{2}} \frac{\partial V_{t r}}{\partial \phi}+2 \frac{\Omega}{\rho \nabla_{\perp}^{2} V_{t r}} \frac{\partial V_{t r}}{\partial \phi},
\end{aligned}
$$

and

$$
\begin{aligned}
\rho \frac{\partial \phi}{\partial t}= & \frac{\hbar q \ln \left(r_{c}\right)}{2 M g\left|\Psi_{T F}\right|^{2}} \frac{\partial \rho}{\partial z} \frac{\partial V_{t r}}{\partial z}+\frac{3 \hbar q \ln \left(\frac{R_{\perp}}{r_{c}}\right)}{4 M g\left|\Psi_{T F}\right|^{2}} \frac{\partial V_{t r}}{\partial \rho} \\
& +\frac{\hbar q \ln \left[\frac{1}{r_{c}} \sqrt{\frac{\rho}{\rho\left(\partial_{z} \phi\right)^{2}-\partial_{z}^{2} \rho}}\right]}{2 M}\left[\frac{\partial^{2} \rho}{\partial z^{2}}-\rho\left(\frac{\partial \phi}{\partial z}\right)^{2}\right] \\
& -2 \frac{\Omega}{\nabla_{\perp}^{2} V_{t r}} \frac{\partial V_{t r}}{\partial \rho} .
\end{aligned}
$$

From these nonlinear equations, the complete behavior of the vortex line can be determined; however, this paper will only look at some general and simple examples of these equations.

\section{WAVE INVESTIGATIONS}

\section{A. Qualitative comments}

These equations give deterministic properties of a slightly perturbed vortex in a BEC. However, before looking at a special case of this, it is worth looking at qualitative behavior from Eq. (82).

The vortex contains angular momentum in $\hat{\mathbf{z}}$. Therefore, as suggested by Fetter [7], the vortex can be considered as a gyroscope spinning in $\hat{\mathbf{z}}$, responding to any force by moving perpendicular to it. In Eq. (82), there are three such forces: a trap gradient force $\left[-\nabla V_{t r}\left(\mathbf{r}_{\mathbf{0}}\right)\right]$, a curvature force $(\kappa \hat{\mathbf{n}}, \hat{\mathbf{b}} \approx \hat{\mathbf{z}} \times \hat{\mathbf{n}})$, and a $z$-trap deviation force $\left[\partial_{z} V_{t r}\left(\mathbf{r}_{\mathbf{0}}\right) \hat{\mathbf{t}}\right]$, with each force contributing to the relation's final form.

Similarly, the rotation of the condensate $\boldsymbol{\Omega}$ also creates gyroscopic motion. This rotation causes any point in the trap to move perpendicular to the trap gradient force, and therefore also pushes the vortex line accordingly.

Equation (82) also contains a collection of terms that "amplifies" the velocity of the line in the $\rho$ direction. These terms are additional restoring forces, which attempt to return the system to the the centered straight-line vortex state.

Hence the qualitative behavior depicted by Eq. (82) gives understandable insight into how the vortex line moves; therefore, this indicates that it is practical to use it to quantitatively determine the structure and motion of the vortex line in a condensate.

\section{B. More realistic helical-wave behavior}

As previously mentioned, helical waves can exist on vortex lines in a BEC. Hence it is relevant to consider the behavior of such waves in the presence of a trapping potential. Assuming a cylindrical trap $\left(V_{t r}=M \omega_{\perp}^{2} \rho^{2} / 2\right.$, where $\left.\omega_{z}=0\right)$ and that the helix wave takes the same form used in Sec. VII ( $\rho=a$ and $\left.\phi=k z-\omega t+\phi_{0}\right)$, Eq. (83) is solved and Eq. (84) becomes

$$
\omega=-\frac{3 \hbar q \ln \left(\frac{R_{\perp}}{r_{c}}\right)}{2 M R_{\perp}^{2}\left(1-\frac{\rho^{2}}{R_{\perp}^{2}}\right)}+\frac{\hbar q k^{2} \ln \left(\frac{1}{r_{c}|k|}\right)}{2 M}+\Omega .
$$

This equation shows that the precession helical wave in a cylindrical trap is a combination of the precession of a straightline vortex and the motion of a helical wave in a uniform condensate.

In a numerical simulation by Simula et al. [29,30], it was shown that the dispersion relation of large-wavelength helical waves in a cigar-shaped trap could be represented as

$$
\omega=\omega_{0}+\frac{\hbar q k^{2} \ln \left(\frac{1}{r_{c}|k|}\right)}{2 M}+\Omega,
$$

where $\omega_{0}$ is a constant precession frequency. The similarity of these two equations is striking, and upon plotting (Fig. 3), the similarities are still visible (in the change to $\omega$ to the changing particle number); however, the specific values of the constant vary by about $\pm 0.1\left(\omega / \omega_{\perp}\right)$ to the numerical value.

This deviation is not surprising. The constant precession of Eq. (85) is that of a purely straight-line vortex. This suggests that Eq. (85) is representative of pancake-shaped traps where such vortices occur. Simula's work, however, is in a cigar-shaped trap and therefore the vortex line, without wave perturbations, tends to bend, potentially changing its precession. Therefore, to accurately depict this behavior, the trapping of the condensate in $z$, and how it distorts the vortex, needs to be considered.

\section{PREVIOUS SIMILAR WORK}

A similar derivation has been carried out by Svidzinsky and Fetter [25] with the aim of determining the normal waves that a vortex line can support. This work performed a similar 


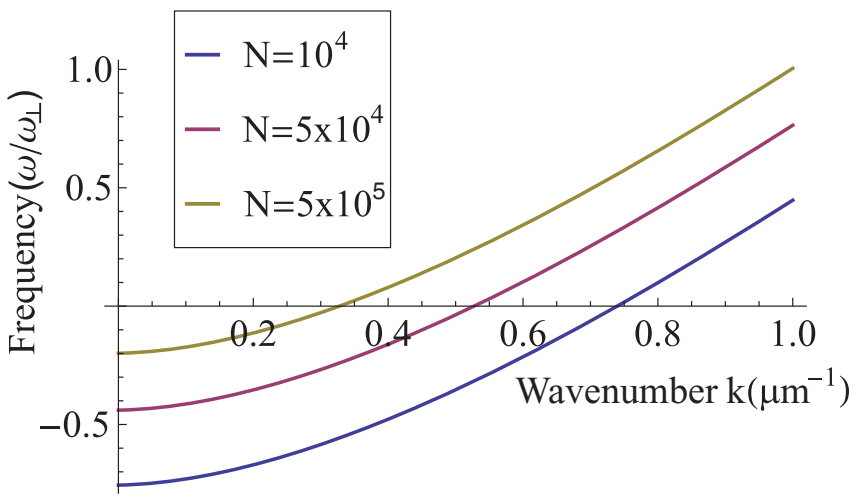

FIG. 3. (Color online) Plots of the dispersion relation, given by Eq. (85), for different particle number $(N)$ for a condensate of ${ }^{87} \mathrm{Rb}$ atoms, with trapping frequencies $\omega_{z}=2 \pi \times 11.8 \mathrm{~Hz}$ and $\omega_{\perp}=2 \pi \times 98.5 \mathrm{~Hz}$, and vortex core radius $r_{c}=0.13 \mu \mathrm{m}$. The upper line in the plot corresponds to $N=5 \times 10^{5}$, the middle line corresponds to $N=5 \times 10^{4}$, and lower line corresponds to $N=10^{4}$.

matched asymptotic expansion to determine a relation for the vortex line's velocity.

During this derivation, an extra term appeared in the outer equations that was related to the transformation into the local coordinates of the vortex line, the specifics of which were not explained in the paper. This gave an outer solution of

$$
\Phi=-e^{\frac{\kappa x}{2}} K_{0}\left(\rho \sqrt{\frac{\nabla_{\perp}^{2} V_{t r}}{2 g\left|\Psi_{T F}\right|^{2}}+\frac{\kappa^{2}}{4}}\right),
$$

which was approximated to be

$$
\Phi=\ln \left(\frac{e^{c}}{\sqrt{2}} \rho \sqrt{\frac{1}{R_{\perp}^{2}}+\frac{\kappa^{2}}{4}}\right),
$$

for small $\rho$. This form is almost identical to that derived in this paper, having effectively ignored the additional exponential in their outer solution.

Then, to compare the outer and inner solutions, a pseudo $\Phi$ was created for the inner phase solution. This pseudo $\Phi$ was linked with the inner-solution phase $S$ through the same definition of $\Phi$ used in Sec. V, but ignored some terms, of comparable order to those kept,

$$
\Phi \approx\left[1+\frac{x}{2}\left(\frac{\left|\nabla_{\perp} V_{t r}\right|}{g\left|\Psi_{T F}\right|^{2}}+\kappa\right)\right] \ln (E \rho)+\frac{M V_{0}}{\hbar q} x .
$$

It is worth noting that the largest term of this inner $\Phi$ is $x \ln (\rho)$, while the largest term of the outer $\Phi$ is $\ln (\rho)$. This indicates an inconsistency in the expansion of the outer solution [the $\mathrm{e}^{\frac{\kappa x}{2}}$ in Eq. (87) allowing the outer to also expand to $x \ln (\rho)]$.

Through matching these $\Phi$ s, the velocity condition found was

$$
\begin{aligned}
\mathbf{V}\left(\mathbf{r}_{\mathbf{0}}\right)= & -\frac{q \hbar \hat{\mathbf{t}} \times \nabla V_{t r}}{2 M g\left|\Psi_{T F}\right|^{2}} \ln \left(|q| r_{c} \sqrt{\frac{1}{R_{\perp}^{2}}+\frac{\kappa^{2}}{4}}\right) \\
& -\frac{q \hbar \kappa \hat{\mathbf{b}}}{2 M} \ln \left(|q| r_{c} \sqrt{\frac{1}{R_{\perp}^{2}}+\frac{\kappa^{2}}{4}}\right)+2 \frac{\nabla V_{t r} \times \boldsymbol{\Omega}}{\nabla^{2} V_{t r}} .
\end{aligned}
$$

In deriving this, the effect of $\hat{\mathbf{t}}$ deviating from $\hat{\mathbf{z}}$ was not considered, but later implemented through the $\hat{\mathbf{t}} \times \nabla V_{t r}$ term. This procedure gives the same $\partial_{z} V_{t r}$ structure seen in Eq. (82) but also adds an additional artifact that if not balanced, will create a line velocity in $\hat{z}$ [specifically it would give $V_{z}\left(\mathbf{r}_{0}\right) \propto$ $\left.x_{0} \partial_{y} V_{t r}\left(\mathbf{r}_{0}\right)-y_{0} \partial_{x} V_{t r}\left(\mathbf{r}_{0}\right)\right]$. However, in their derivation, it was assumed that such motion in $\hat{z}$ did not occur, and when the derivation is repeated allowing the line at any point to deviate slightly from $\hat{z}$, as done here, this condition is not found.

A comparison of Eqs. (90) and (82) shows that there are distinct similarities and differences. The terms related to the system's rotation $(\boldsymbol{\Omega})$ are identical in both cases, the curvature $(\kappa \hat{\mathbf{b}})$ terms both multiply logarithms that also depend on curvature, and there are terms relating the vortex line's structure and the trap gradient $\left(\nabla V_{t r}\right)$. However, the constants around these terms are different, the logarithms vary in form from term to term, and there are no terms with the $\hat{\rho} \otimes \hat{\rho}$ structure in Eq. (90). These $\hat{\rho} \otimes \hat{\rho}$ terms particularly are worth a mention. They arise from the derivatives of $\ln (\rho)$ when obtaining the gradient of the inner phase [Eq. (62)], and are smaller than the logarithmic terms, being constant, giving an essentially different structure up to logarithmic accuracy. This is easily shown when considering the curvature term. The curvature term of Eq. (82) has two components: the logarithm term, being proportional to $\kappa \ln (1 / \kappa)$, and the $\hat{\rho} \otimes \hat{\rho}$ term, being proportional to $\kappa$; the latter obviously being the smaller term.

Furthermore, note that though the solution presented in this paper [Eq. (82)] still has an arbitrary constant yet to be determined, the solution cannot turn into Eq. (90). The arbitrary constant has components in $\hat{x}$ and $\hat{y}$ but not $\hat{z}$, as there were no differential equations relating to the $\hat{z}$ direction to solve. So although the constant can depend on $x_{0}, y_{0}, \partial_{x} V_{t r}\left(\mathbf{r}_{0}\right)$, and $\partial_{y} V_{t r}\left(\mathbf{r}_{0}\right)$, the constant is unable to apply the condition required to rewrite it into the form of Svidzinsky and Fetter's results.

Hence because of these differences, Eqs. (90) and (82), though able to give similar wave results within certain limits, can differ significantly. For example, both equations replicate the long-wavelength helical-wave dispersion relation, given by Eq. (76), but when looking at helical waves in a condensate with trapping in $\rho\left(V_{t r}=M \omega_{\perp}^{2} \rho^{2} / 2\right)$, Eq. (90) gives a different constant precession frequency $\left(\omega_{0}\right)$ of

$$
\omega_{0}=-\frac{\hbar}{2 \mu} \ln \left(\frac{R_{\perp}}{r_{c}}\right),
$$

where $\mu$ is the chemical potential of the system.

The $\omega_{0}$ obtained in Eq. (85) is $50 \%$ larger than the $\omega_{0}$ obtained from Eq. (90) [Eq. (91)] under the same conditions. This difference gives a larger deviation between Eq. (91) and the $\omega_{0}$ found by Simula et al. in their numerical simulations than found in this paper.

In a later review by Svidzinsky and Fetter [31], the dynamics of a straight vortex in a disk-shaped condensate was discussed. In this discussion, the describing equation for such lines was shown to be similar to Eq. (90), when $\kappa=0$ and $\hat{\mathbf{t}}=\hat{\mathbf{z}}$, with the difference between the results being a factor of $3 / 2$ multiplying the $\ln \left(R_{\perp} / r_{c}\right)$ term. This behavior is therefore not accounted for within Eq. (90). However, 
Eq. (82) has such a $3 / 2$ multiplying it's $\ln \left(R_{\perp} / r_{c}\right)$ term and so can describe the behavior in this limit (for $\kappa=0$ and $\hat{\mathbf{t}}=\hat{\mathbf{z}}$.

\section{CONCLUSIONS AND FUTURE DIRECTIONS}

Through this derivation, a robust relation for the motion and shape of a vortex line in a BEC was determined [Eq. (82)]. This relation was found by determining the behavior of the condensate near and far from the vortex core separately, and ensuring that these two solutions matched in the overlapping region.

This relation was shown to describe well the local vortex's behavior, and could be transformed into a set of differential equations that describes the vortex's overall structure and motion [Eqs. (83) and (84)]. Using a simple calculation, these equations reflected wave behavior seen numerically.

This paper only briefly explored the behavior of Eqs. (83) and (84), and so further investigation needs to be performed, looking at different vortex line structures and trapping geometries. However, before this can be done, an analytic description of how the trapping in $z$ affects the line's behavior is needed to calibrate the final term in Eq. (82).

\section{ACKNOWLEDGMENTS}

The authors would like to thank Alexander Fetter and Tapio Simula for useful discussions and general advice.
[1] M. R. Matthews, B. P. Anderson, P. C. Haljan, D. S. Hall, C. E. Wieman, and E. A. Cornell, Phys. Rev. Lett. 83, 2498 (1999).

[2] B. P. Anderson, P. C. Haljan, C. E. Wieman, and E. A. Cornell, Phys. Rev. Lett. 85, 2857 (2000).

[3] K. W. Madison, F. Chevy, W. Wohlleben, and J. Dalibard, Phys. Rev. Lett. 84, 806 (2000).

[4] F. Chevy, K. W. Madison, and J. Dalibard, Phys. Rev. Lett. 85, 2223 (2000).

[5] V. Bretin, S. Stock, Y. Seurin, and J. Dalibard, Phys. Rev. Lett. 92, 050403 (2004).

[6] J. Low Temp. Phys. 161, 574 (2010).

[7] A. L. Fetter, Rev. Mod. Phys. 81, 647 (2009).

[8] R. P. Feynman, Prog. Low Temp. Phys. 1, 17 (1955).

[9] C. Pethick and H. Smith, Bose-Einstein Condensation in Dilute Gases (Cambridge University Press, Cambridge, UK, 2002).

[10] L. P. Pitaevskii, Sov. Phys. JETP 13, 451 (1961).

[11] E. P. Gross, Nuovo Cimento 20, 454 (1961).

[12] A. A. Svidzinsky and A. L. Fetter, Phys. Rev. Lett. 84, 5919 (2000).

[13] P. O. Fedichev and G. V. Shlyapnikov, Phys. Rev. A 60, R1779 (1999).

[14] S. A. McGee and M. J. Holland, Phys. Rev. A 63, 043608 (2001).

[15] A. Aftalion and T. Riviere, Phys. Rev. A 64, 043611 (2001).

[16] J. J. García-Ripoll and V. M. Pérez-García, Phys. Rev. A 64, 053611 (2001).
[17] P. Rosenbusch, V. Bretin, and J. Dalibard, Phys. Rev. Lett. 89, 200403 (2002).

[18] M. Modugno, L. Pricoupenko, and Y. Castin, Eur. Phys. J. D 22, 235 (2003).

[19] F. Zambelli and S. Stringari, Phys. Rev. Lett. 81, 1754 (1998).

[20] P. C. Haljan, B. P. Anderson, I. Coddington, and E. A. Cornell, Phys. Rev. Lett. 86, 2922 (2001).

[21] W. Thomson (Lord Kelvin), Philos. Mag. 10, 155 (1880).

[22] V. Bretin, P. Rosenbusch, F. Chevy, G. V. Shlyapnikov, and J. Dalibard, Phys. Rev. Lett. 90, 100403 (2003).

[23] B. Y. Rubinstein and L. M. Pismen, Physica D 78, 1 (1994).

[24] L. M. Pismen and J. Rubinstein, Physica D 47, 353 (1991).

[25] A. A. Svidzinsky and A. L. Fetter, Phys. Rev. A 62, 063617 (2000).

[26] F. Verhulst, Methods and Applications of Singular Perturbations: Boundary Layers and Multiple Timescale Dynamics (Springer, Berlin, 2005).

[27] W. Kühnel, Differential Geometry: Curves - Surfaces - Manifolds (American Mathematical Society, Providence, RI, 2002).

[28] J. R. Anglin, Phys. Rev. A 65, 063611 (2002).

[29] T. P. Simula, T. Mizushima, and K. Machida, Phys. Rev. Lett. 101, 020402 (2008).

[30] T. P. Simula, T. Mizushima, and K. Machida, Phys. Rev. A 78, 053604 (2008).

[31] A. L. Fetter and A. A. Svidzinsky, J. Phys. Condens. Matter 13, R135 (2001). 